\title{
Using Cognitive Radio Principles for Wireless Resource Management in Home Networking
}

\author{
E. Meshkova ${ }^{\star}$, Z. Wang ${ }^{\dagger}$, J. Nasreddine ${ }^{\star}$, D. Denkovski ${ }^{\dagger \dagger}$, C. Zhao ${ }^{\dagger}$, K. Rerkrai ${ }^{\star}$, \\ T. Farnham ${ }^{\diamond}$, A. Ahmad ${ }^{\dagger}$, A. Gefflaut ${ }^{\dagger}$, L. Gavrilovska ${ }^{\dagger \dagger}$, P. Mähönen ${ }^{\star}$ \\ * Institute for Networked Systems, RWTH Aachen University, Germany \\ $\dagger$ European Microsoft Innovation Centre, Germany \\ $\diamond$ Toshiba European Research Laboratory, UK \\ ${ }^{\dagger}$ Faculty of Electrical Engineering, Ss. Cyril and Mehtodius, Skopje, Macedonia \\ email: eme@inets.rwth-aachen.de
}

\begin{abstract}
The demand for higher data rates, capacity and better quality-of-service is constantly growing for home networks. Therefore, there is a pressing need for efficient use of wireless network resources. In this context, the application of cognitive radio principles that enable network nodes to characterize their environment and control their resources based on the acquired knowledge, is the prominent solution for next generation home networks. In this paper we present an architecture and a prototype implementation based on these principles. The proposed system is able to autonomously optimize the performance of network nodes in a dynamic environment according to the goals, restrictions and policy regulations formulated by network stakeholders. The obtained results show the momentous and suitability of the cognitive framework for home networking.
\end{abstract}

\section{INTRODUCTION}

The popularity of wireless communications systems for home networking is rapidly growing along with the user demand for diverse high quality telecommunications services. This results in a dense wireless environment with fast channel variations and uncontrolled interference, which highly complicates the design of wireless systems.

In particular, a notable trend in Consumer Electronic (CE) is the increasing number of Wi-Fi enabled devices, like cameras, TVs and other digital media appliances. In 2012, the shipments of CE devices and mobile handsets equipped with WLAN interfaces is forecasted to reach nearly one billion units [1]. The increasing adoption of Wi-Fi creates new usage models, like wireless content sharing and streaming between home media appliances. However, current network management solutions do not assist non-professional users to efficiently setup and maintain IEEE 802.11 networks. For example, networks are often configured to operate on a single channel and to route the traffic through an access point (AP). This leads to non-optimal allocation of wireless resources, which results in network overloading. Therefore, there is a pressing need for a system that autonomously manages wireless home networks in accordance to changing user needs and environment dynamics.

Traditional highly efficient Radio Resource Management (RRM) systems [2] typically employ centralized architectures, characterized by high complexity and low flexibility, and are much more suitable for cellular environments than home networks. The latter are smaller, less cooperative and more aggressive in terms of interference and quality of service requirements. In this context, Cognitive Radio (CR) [3] is a promising alternative paradigm to traditional planning and RRM techniques.

Cognitive nodes are aware of user preferences and environment characteristics, that are obtained, for e.g, through policies, sensing and cooperation. They are able to autonomously optimize network performance by flexibly employing various resource management techniques, such as dynamic spectrum access and cross-layer optimization. However, most of the research on CR is focusing on only Dynamic Spectrum Access (DSA) methods [4]. The general concept of cognitive radio is only considered in few works [5], [6], where no practical implementation has been produced.

In this paper we suggest and implement a system architecture that aims at autonomous optimization of resource usage and performance in home wireless environments. The framework utilizes the Mitola's cognitive radio concept, and is named after [6], a Home Cognitive Resource Manager (HCRM). The system consists of cognitive autonomous agents residing on each node. These agents are capable of both cooperative and non-cooperative actions allowing for both centralized and decentralized network management. Each agent manages jointly multiple components using different optimization strategies that can be adapted in the run-time. The optimization goals are set by network stakeholders through policies and utilities [7], which express desired Quality-ofExperience (QoE) levels and state regulatory, hardware and other system constraints. The framework strives to maximize performance of a home network across the whole protocol stack using traffic redirection, dynamic spectrum access methods, and cross-layer optimization. The maximization process involves application-layer configuration, as well as traffic and channel shaping. The system operates using both low-layer and high-layer performance metrics to enable fast, reliable and accurate reaction to changes in the network. The prototype of the HCRM is implementated on the Windows XP platform using low cost Commercial Off-the-Shelf (COTS) hardware.

The paper is further organized as follows. In Section II 
we describe the major challenges faced when designing a cognitive framework to home networking. In Section III we describe the architecture of the autonomous home agent. In Section IV we provide the sample results from our prototype implementation. Section V concludes the paper.

\section{DESIGN REQUiREMENTS}

The HCRM system is a part of the network control plane and its main objective is to maximize the performance of a home environment in terms of metrics that reflect the satisfaction of the network stakeholders. Typical stakeholders of a home network are users and providers. Their dynamically changing objectives should be conveyed to the system maximally accurately in order to achieve the best optimization results. The system must meet additional constraints imposed, for e.g., by equipment capabilities and regulation bodies. Therefore, the framework has to be extensible and flexible, and it requires generic interfaces to interact with network's configurable components ${ }^{1}$. For example, a common interface to various wireless technologies, like WLAN or UMTS, enables low-layer plug-and-play reconfigurability of a system [8].

Autonomous home agents need to solve the network performance optimization problem in a distributed manner. The obtained benefits of their solution must also balance out signaling overhead and the level of instability and disturbance imposed on the stakeholders. They should also be capable of resolving problems with partial knowledge, or even no knowledge, of the network environment. The input for the system could be obtained both locally from different layers of a communication stack and distantly using information from other, primarily cooperative, nodes. The home architecture should resolve conflicts arising due to the use of autonomous agents with potentially competitive optimization goals and minimize the levels of their mutual interference. Additional conflicts can arise from solving optimization problems at different time scales that range from few microseconds (PHY layer) to few seconds and even minutes (application layer).

\section{ARCHITECTURE}

\section{A. Design Principles}

The architecture of the HCRM is based on two major design principles, which allow it to meet the requirements outlined in the previous section. These are the Marr's basic abstraction of a cognitive system and the feedback control loop.

The three complementary levels at which a cognitive system may be described, formulated by Marr [9], are the goal, method and implementation levels that explain "how the goal is reached with a method that is allowed by the means". In the HCRM we use policies and utilities to state the optimization goal for the system. As the method for our architecture we have chosen the feedback control loop design principle, which allows for scalable, distributed and flexible design of a home environment. The means of HCRM to pursuit the performance

\footnotetext{
${ }^{1}$ In this article we understand under components individual configurable network protocols, middleware and other modules used in networking.
}

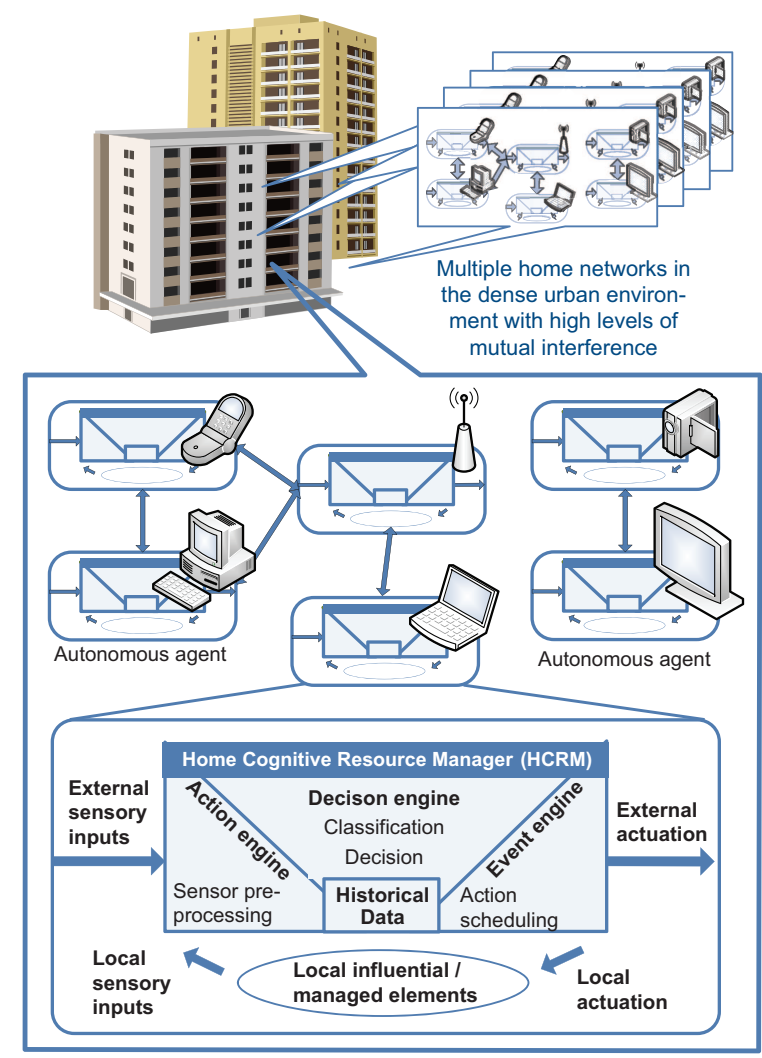

Fig. 1. Conceptual design of the Home Cognitive Resource Manager is based, among others, on the modified notion of the feedback control loop.

maximization task is to operate on multiple configurable components. Additionally, several optimization algorithms can be used complimentary or exchangeably in our system to enable more efficient network resource management. For example it can be a decision tree, that is used in our prototype, or more sophisticated methods based on, for example, convex optimization and machine learning.

Feedback control loop principle, see Figure 1, is popular in the domains of cognitive radio networking [3], autonomous computing [10], as well as in control theory and machine learning. Each autonomous agent executes a variant of feedback loops, for which inputs are the sensory data from network performance gathered both locally and externally on all layers of the protocol stack. These inputs along with the historical data is used then to reach the next valid configuration of a node that is likely to lead to the best network performance. This solution is then scheduled through series of actuation actions that are deployed at different time scales and dictated by the timing constraints of individual optimized components.

\section{B. Architecture of the Autonomous Agents}

The architecture of the autonomous HCRM agent is shown in Figure 2. It includes four major blocks: the interfaces, the middleware, the pre/post-processing and the decision modules. The interfaces, see Section III-E, convey sensory input and actuation data between the whole communication stack and 


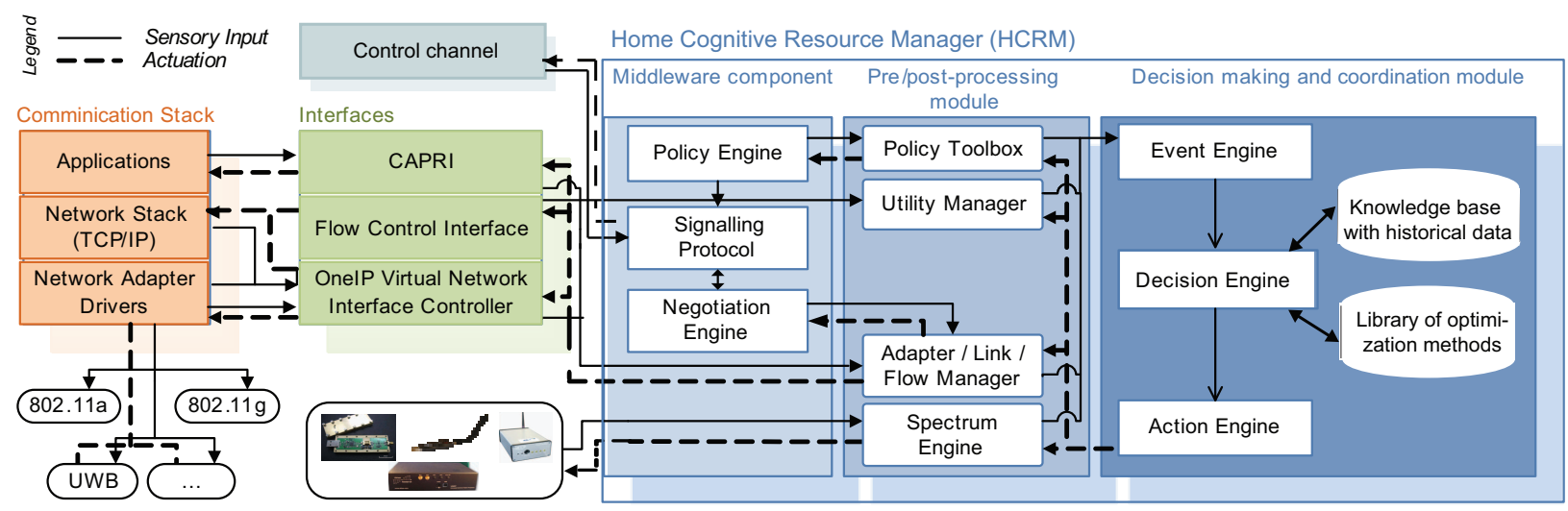

Fig. 2. Overview of the architecture of the Home Cognitive Resource Manager that includes three basic system modules (decision, middleware and pre/post-processing), and interacts with the communication stack via specially developed interfaces.

HCRM. Monitoring of both low and high layer Key Performance Indicators (KPIs) allows our framework to react to the changes in the network performance in a rapid and accurate manner. This also helps in avoiding costly actions when they are unnecessary. For instance, due to hardware constraints in our current implementation, frequency hopping can go to the orders of seconds. It is therefore, detrimental to execute this operation too often. The low-layer KPIs, like driver load and packet error rate, allow quick detection of unfavorable channel conditions. However, if high-layer utility-based performance assessment, formulated using application- and transport-layer metrics, indicates that the user is still satisfied with the quality of an application, switching to a slightly less congested channel is not likely to bring significant performance gains. Therefore this action will not be taken. However, this decision could be different for another application, which is more vulnerable to higher packet losses.

The middleware block handles communication with other HCRM agents, and interacts with the policy framework. The communication with other HCRM agents is done through the control channel, which can be realized both using in-band and out-of-band signaling. The negotiation engine interacts with the cooperative HCRM agents to agree on communication parameters, request their data, and notify on actions to be performed. The control channel is also utilized by the policy framework of the HCRM, which is further described in Section III-C. The post/pre-processing module handles both periodic and asynchronous commands, as well as data exchange between the decision module and the network stack, spectrum analyzers, and the policy framework, via the Adapter/Link/Flow (ALF) manager, the utility manager, the spectrum engine and the policy toolbox respectively. The ALF manager also detects new applications and initializes the configuration of their data flows. It automatically manages wireless interfaces on each node, e.g. detect their failures or appearance, and provides seamless handover between them. The decision module, described further in Section III-D, optimizes performance of a home agent through configuration of its components or the components residing on other network nodes.

\section{Policy Framework in HCRM}

Policies represent a set of rules, constraints and preferences imposed by different network stakeholders, including regulatory bodies. They govern the behavior of network devices by restricting the space of available solutions for HCRM, i.e. policies limit the amount of configuration states available for an optimizer [11]. Additionally, policy preferences are used as part of utility functions to express objectives of the stakeholders more fully.

The implementation of the policy-based cognitive networking concept for home environment includes two types of policies. The spectrum and connectivity policies are used to control access and usage of available resources in the home environment such as frequency bands. The preferential policies dictate the priorities and preferences of different users and applications. Policies can operate constantly or during a pre-defined time period, depending on, for e.g., time of the day. The HCRM policy framework operates on classes of home network users and devices that can be dynamically changed. This allows to reason and prioritize on groups of users/terminals and resolve conflicts of interest between them.

The three main components that constitute the policy framework [11] are shown in Figure 3. The policy server is the repository of all policies in the home policy system. It can be updated by both local and external bodies, e.g. home users and spectrum regulators respectively. The policy engine, realized using [12], is a device component that reasons on the dedicated set of policies when required by the HCRM. The policy handling toolbox is a part of a HCRM home agent that interacts with the policy engine.

\section{Decision Making Block}

The decision making and coordination module of the HCRM realizes the core of the feedback control loop. Based on inputs from different layers, nodes, and maintained historical data, it estimates the current performance of the network, identifies the most likely cause of a problem and schedules actions to mitigate it. This HCRM module includes three blocks the event, decision and action engines. The main goal of 


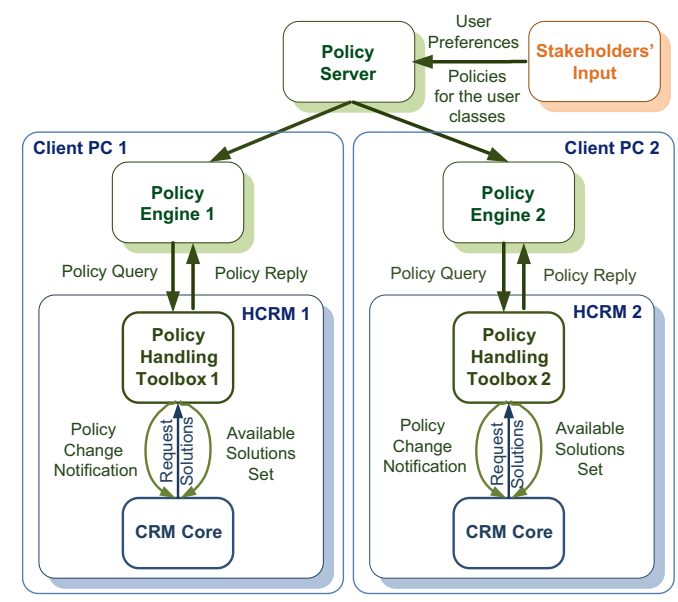

Fig. 3. The HCRM policy framework includes the central policy server, as well as the policy engine and the policy toolbox residing locally.

the event and action engines is the propagation of information between the decision engine and the rest of the HCRM. These engines signal the data asynchronously, allowing for multiple optimization algorithms to be executed in parallel at different time-scales (including close-to-runtime). Thus they enable realization of multiple control loops in the HCRM.

The decision engine is modular in design and is capable of loading different optimization algorithms to vary its function. For example, in case of a policy update a simple decision tree can be executed to check the validity of the current agent configuration. However, more complex and time-demanding machine learning mechanisms might be needed to guide the cross-layer optimization process in large solution space [13]. The decision engine also performs a limited conflict resolution that might arise during asynchronous handling of events.

The functionality of the decision engine is split into two parts classification and decision making. The classification module is required to determine the environment/conditions in which the network is operating, for e.g., presence of a hidden terminal, a link overload, or high interference. Based on sensory inputs and conducted classification the decision is made on the optimization algorithm to be executed, which results in the list of actions for the HCRM.

\section{E. Interfaces and the Utility Abstraction in HCRM}

To interact with configurable network components the HCRM uses the virtual driver wrapper called OneIP, the flow control and Common Application Requirement Interface (CAPRI). The OneIP wrapper abstracts network interfaces and corresponding communication parameters of the PHY and MAC layers ${ }^{2}$. The flow control interface handles information exchange on network and transport layer statistics and configurations of parameters.

The Quality-of-Experience (QoE) expected by individual data flows, applications, nodes and the overall network are

\footnotetext{
${ }^{2}$ Other example of a generic interface that can be used to abstract the same low-layer functionality is a Unified Link Layer API (ULLA) [14].
}

expressed using utilities. These functions are used by the decision module to achieve utility-based optimization goals. CAPRI interface [7] allows the applications to express and dynamically update their objectives as combination of transportlayer KPIs, such as throughput, delay or jitter, to the utility manager of the HCRM agent. The latter analyzes the individual utility functions, formulates the overall objective function and calculates instantaneous utility values to be used during the decision phase. Alternatively, if these functions are not provided or legacy application are executed, default utility specifications are used.

\section{IMPLEMENTATION AND SAMPLE RESULTS}

We have implemented the home cognitive resource manager, demonstrated in [15], on the Windows platform using low cost COTS hardware such as Atheros WLAN 802.11a/g cards and Metageek Wi-Spy spectrum analyzers [16]. The prototype provides wireless connectivity in an optimized way with enhanced capability to withstand interference and contention. The system allows to dynamically establish adaptable pointto-point connections between HCRM nodes while maintaining the connection to the AP (Access Point). We use the AP-based communication as the control channel, which is enabled by the installation of a second wireless interface card on each node. In our scenario we use IEEE $802.11 \mathrm{~g}$ cards. In the presented scenario we run a network of four nodes organized into pairs that transmit variable multimedia and download data. The parameters adapted in this scenario are central channel frequency, channel width, traffic shaping using ClassBased Queueing (CBQ) and traffic redirection. The continuous performance of the network during re-configuration phases is ensured through using the control channel as the relay point. Currently the HCRM decision engine invokes a decision tree algorithm for network optimization. The influential KPIs that are monitored as part of the feedback control are throughput, link load, packet error rate, RSSI, spectrum occupancy power and, of course, utilities. Additionally, the nodes limitedly exchange their configuration data for more efficient decision making, e.g. to find a common transmission channel. Figure 4 shows the annotated graphs of goodput, utilities and the waterfall spectrogram of energy distribution over $2.4 \mathrm{GHz}$ band obtained when running the scenario $^{3}$. The figure also displays the utilities of separate data flows ${ }^{4}$. Our experiments show that the HCRM agents are successful in autonomously handling dynamic variations in external interference patterns and changes in the mixture of applications executed by the nodes, without breaking any connections at the application level and maintaining high levels of the overall network utility.

\footnotetext{
${ }^{3}$ Channel 1 is used as the control channel and, therefore, it is forbidden through policies on establish ad-hoc links on the involved frequencies.

${ }^{4}$ On the right size of Figure 4 individual utilities of the data flows are shown. The overall normalized network utility, due to the applications priorities, is $U_{\text {netw }}=U_{\text {pair } 1}+U_{\text {pair } 2}=\left(\left(U_{H D}+6 U_{D}+3 U_{U D P}\right) / 10+U_{U D P}\right) / 2$, where $U_{H D}$ is the utility of high definition video over TCP in form of the step function, $U_{U D P}$ is the utility of the streaming video of UDP with maximum datarate of $2 \mathrm{Mbps}$, and $U_{D}$ is the logarithmic utility function of the download.
} 


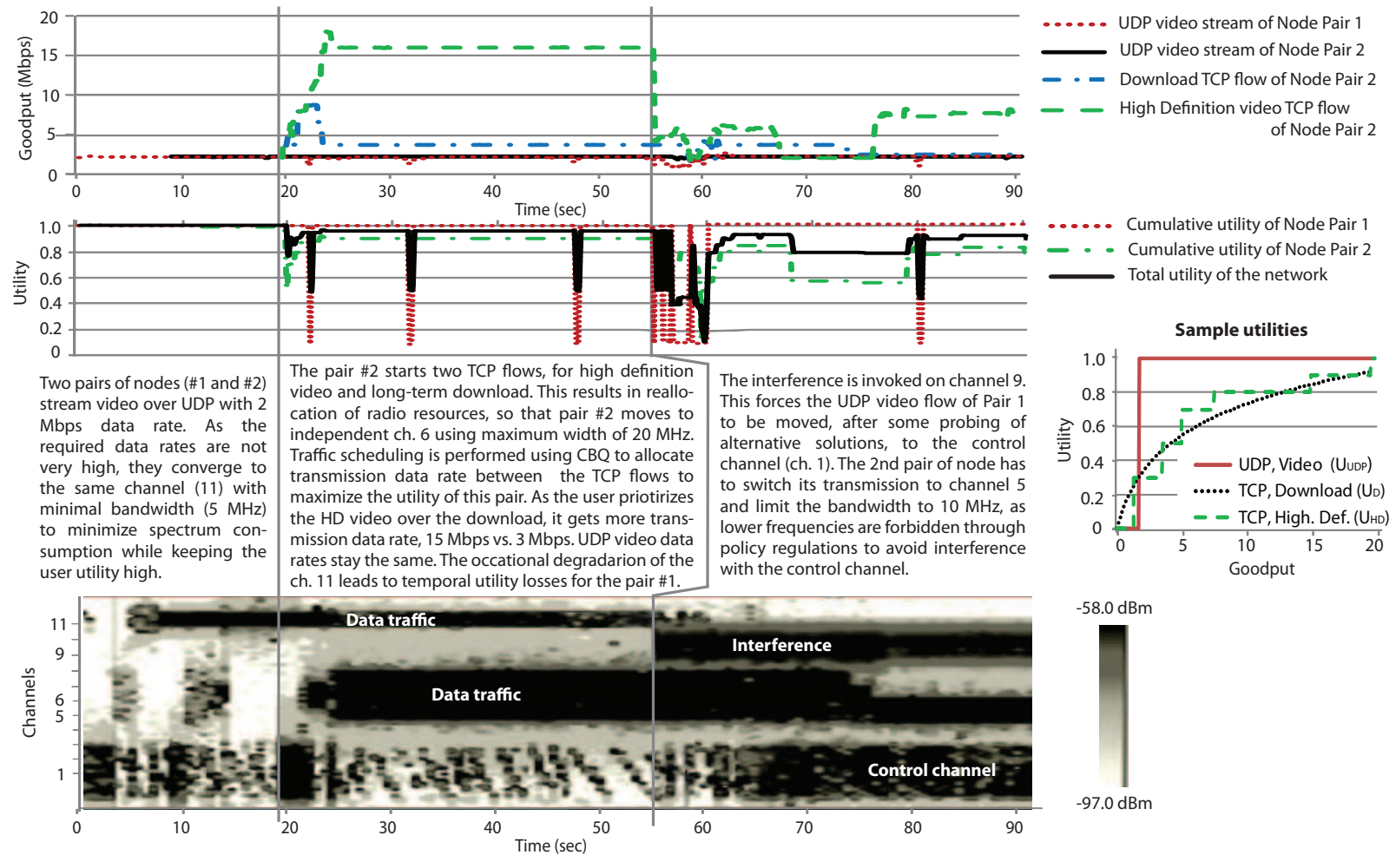

Fig. 4. Performance of four node network in the dynamic environment with changing number of applications. The figure displays (from top) the goodput achieved by the data flows; the overall network utility and utility functions of the nodes; the waterfall spectrogram of power distribution in $2.4 \mathrm{GHz}^{3,4}$.

\section{CONCLusions}

In this paper we suggested the architecture of the Home Cognitive Resource manager and provided its preliminary evaluation using a developed prototype. The HCRM and its home agents are able to autonomously optimize the network performance in a dynamic environment, based on the goals and restriction provided by network stateholders. The system is able to automatically establish and maintain point-to-point connections between home nodes and configure them using both low- and high-layer communication stack parameters in the run-time. In the future, we plan to explore different machine-learning optimization techniques that can be utilized by the HCRM, and study the cross-layer optimization possibilities and dependencies in wireless networks.

\section{ACKNOWLEDGMENT}

Authors would like to thank EU (ARAGORN project [17]), and EM, JN, KR, PM the German Research Foundation (through UMIC) for partial financial support. We thank the collaborators in the ARAGORN consortium for their contributions, especially B. Motz, V. Atanasovski and M. Petrova.

\section{REFERENCES}

[1] [online] Intel My WiFi Technology Tech Brief, http://download.intel.com/network/connectivity/products/wireless/ Intel_R_My_WiFi_Technology_Tech_Brief.pdf, [Visited: 26/05/2010].

[2] J. Zander and S. L. Kim, Radio Resource Managment for Wireless Networks. Norwood, MA: Artech House, 2001.
[3] J. Mitola and G. Maguire, "Cognitive radio: making software radios more personal," IEEE Personal communications, vol. 6, no. 4, pp. 13$18,1999$.

[4] I. F. Akyildiz et.al, "Next generation/dynamic spectrum access/cognitive radio wireless networks: a survey," Computer Networks, vol. 50, no. 13, pp. $2127-2159,2006$.

[5] R. W. Thomas et.al, "Cognitive networks: adaptation and learning to achieve end-to-end performance objectives," IEEE Communications Magazine, vol. 44, no. 12, pp. 51 -57, Dec. 2006

[6] M. Petrova et.al, "Cognitive wireless networks: Your network just became a teenager," in IEEE INFOCOM, poster paper, Barcelona, 2006.

[7] J. Riihijärvi et.al, "Extending Policy Languages with Utility and Prioritization Knowledge: The CAPRI approach," in Proc. of IEEE DySPAN, Singapore, April 2010.

[8] J. Nasreddine et. al, "An Implementation of Cognitive Resource Management on LTE Platform," in Proc. of PIMRC, September 2010.

[9] D. Marr, "Vision: A Computational Investigation into the Human Representation and Processing of Visual Information," 1982.

[10] A. Ganek and T. Corbi, "The dawning of the autonomic computing era," IBM Systems Journal, vol. 42, no. 1, pp. 5-18, 2003.

[11] D. Denkovski et.al, "Novel Policy Reasoning Architecture for Cognitive Radio Environments," in Proc. of GLOBECOM, Miami, USA, 2010.

[12] D. Elenius et.al, "CoRaL-Policy Language and Reasoning Techniques for Spectrum Policies," in Proc. of POLICY, 2007, pp. 261-265.

[13] E. Meshkova et.al, "Exploring Simulated Annealing and Graphical Models for Optimization in Cognitive Wireless Networks," in Proc. of IEEE Globecom, Honolulu, USA, November 2009.

[14] M. Sooriyabandara et. al, "Unified Link Layer API: A generic and open API to manage wireless media access," Computer Communications, vol. 31, no. 5, pp. 962-979, 2008.

[15] V. Atanasovski et.al, "Cognitive Radio for Home Networking," in Proc. of IEEE DySPAN, April 2010.

[16] [Online] MetaGeek Wi-Spy $2.4 \mathrm{GHz}$ spectrum homepage, http://www.metageek.net/ [Last visited: 6/06/2010].

[17] [Online] ICT ARAGORN homepage, http://www.ict-aragorn.eu/ [Last visited: $25 / 05 / 2010]$. 